\title{
Stellar or Non-Stellar Light? Determining Near-Infrared Contamination in Low Mass X-ray Binaries
}

\author{
Dawn M. Gelino*, Christopher R. Gelino ${ }^{\dagger}$ and Thomas E. Harrison** \\ *NASA Exoplanet Science Institute, California Institute of Technology, 770 S. Wilson Ave. \\ Pasadena, CA 91125 \\ ${ }^{\dagger}$ Spitzer Science Center, Caltech, 1200 E. California Blvd. Pasadena, CA 91125 \\ **New Mexico State University, Las Cruces, NM 88003
}

\begin{abstract}
Low-mass X-ray binary (LMXB) systems are comprised of a low-mass, K or M dwarflike star orbiting a compact object. Stellar black hole masses and their distributions are important inputs for binary evolution and supernova models. Currently, the main limiting factor in determining accurate black hole masses in LMXBs is the uncertainty of the orbital inclination angle due to an unknown amount of contaminating light in the near infrared. If present, this light dilutes the ellipsoidal variations of the low-mass secondary star, and thus gives the appearance of a lower orbital inclination system. It has been generally thought that the near infrared ellipsoidal light curves of these systems were relatively uncontaminated and represented primarily the light from the lowmass secondary star; however, recent disk and jet models have thrust this thinking into question. We combine our data from the Spitzer Space Telescope with our ground-based optical and near infrared data for several LMXBs to characterize and derive the amount of light contaminating the near-infrared ellipsoidal variations of the low-mass secondary star.
\end{abstract}

Keywords: (Stars:) binaries (including multiple): close; (Stars:) binaries: symbiotic; (Stars:) novae, cataclysmic variables; Stars: low-mass; Stars: late-type; Infrared: stars; X-rays: binaries

PACS: 95.55.Fw; 95.85.Hp; 95.85.Jq; 95.85.Kr; 97.10.Gz; 97.10.Ri; 97.20.Jg; 97.60.Lf; 97.80.Jp

\section{INTRODUCTION}

Low Mass X-Ray Binaries (LMXBs) are important astrophysical systems. They allow us to examine stellar evolution theory in systems with drastically different initial masses. They allow us to determine compact object masses in order to better understand neutron star evolution and constrain their equations of state, as well as to better define the stellar black hole mass function which has implications for stellar evolution theory. Finally, they allow us to examine accretion onto a black hole, which may give us clues to the inner workings of active galactic nuclei.

LMXBs spend most of their lives in a quiescent state, but every few decades (or less frequently), they display large and sudden X-ray and optical outbursts. These outbursts result from a sudden, dramatic increase in the accretion rate onto the compact object from the accretion disk. While in quiescence, the Roche lobe-filling secondary star is visible and shows ellipsoidal variations. 
TABLE 1. LMXB Observations

\begin{tabular}{|c|c|c|c|c|c|c|}
\hline & Optical & Optical Date & Near IR & Near IR Date & Spitzer IR & Spitzer Date \\
\hline QZ Vul & APO* & $2000 / 2001$ & Palomar $^{\dagger}$ & $10 / 20 / 07$ & IRAC & $10 / 21 / 07$ \\
\hline V518 Per & $\mathrm{APO}$ & $2001 / 2003$ & Palomar & $10 / 20 / 07$ & IRAC & $10 / 17 / 07$ \\
\hline GU Mus & CTIO $^{* *}$ & $2000 / 2001$ & CTIO & 2000 & IRAC & $04 / 08 / 08$ \\
\hline
\end{tabular}

\section{DETERMINING BLACK HOLE MASSES}

In order to determine the mass of a black hole in an LMXB, the following quantities need to be known: the orbital period of the system $\left(\mathrm{P}_{\text {orb }}\right)$, the mass ratio $\left(\mathrm{M}_{2} / \mathrm{M}_{1}\right)$, the secondary star radial velocity semi-amplitude $\left(\mathrm{K}_{2}\right)$, and the orbital inclination angle, $i$. In non-eclipsing systems, $i$ is extremely difficult to measure, however, the amplitude of the secondary star ellipsoidal variations is a strong function of $i$. Ellipsoidal variations result from the tidal and rotational distortions of the Roche lobe-filling star, as well as its nonuniform surface brightness distribution, and can be diluted by other light in the system. This dilution of the variations results in an underestimate of $i$, and an overestimate of $\mathrm{M}_{1}$, the mass of the black hole. Luckily, unless the spectrum of the diluting light is the same as that of the secondary star, each wavelength will be diluted by a different amount, and that amount of dilution can be estimated through simultaneous modeling of the observed variations.

\section{DETERMINING IR CONTAMINATION}

Inbetween outbursts, the accretion disks become cool and optically thin and likely do not emit much. We use observations taken during these quiescent times to determine what fraction of the near-IR light is from an accretion disk or other component in the system. We do this by using the differing slopes between power laws and black bodies in the mid-IR Spitzer bands to model the combined ground and space-based data for three LMXBs: QZ Vul, V518 Per, and GU Mus. See Table 1 for information on the LMXB observations, Figures 1 and 2 for our best fit models and a discussion of their implications, and Table 2 for key properties and modeling results for each of the systems.

\section{CONCLUSIONS}

Two out of the three systems discussed here show IR contamination, and it appears that the level of contamination varies with time. This study showed that simultaneous observations across multiple wavelength regimes are essential to understand what is going on in each system. While the data and models indicate that cool disks do not appear to be a significant source of contaminating light since free-free emission cannot explain the optical data, synchrotron jets do appear to play a role in contaminating the $\mathrm{K}$ - 
TABLE 2. LMXB Parameters and SED Model Results

\begin{tabular}{cccc}
\hline Parameter* & QZ Vul & V518 Per & GU Mus \\
\hline X-Ray Name & GS $2000+25$ & GRO J0422+32 & GRS 1124-68 \\
$\mathrm{P}_{\text {orb }}$ (hours) & 8.26 & 5.09 & 10.38 \\
Spec Type $_{2}$ & $\mathrm{~K} 5 \mathrm{~V}$ & $\mathrm{M} 1 \mathrm{~V}$ & $\mathrm{~K} 4 \mathrm{IV}$ \\
$\mathrm{M}_{2}(\mathrm{M} \odot)$ & $0.32 \pm 0.11$ & $0.46 \pm 0.31$ & $0.94 \pm 0.20$ \\
$\mathrm{R}_{L_{2}}(\mathrm{R} \odot)$ & $0.66 \pm 0.08$ & $0.53 \pm 0.16$ & $1.08 \pm 0.05$ \\
$a(\mathrm{R} \odot)$ & $4.14 \pm 0.13$ & $2.45 \pm 0.24$ & $4.85 \pm 0.15$ \\
$i\left({ }^{\circ}\right)$ & $64 \pm 1.5$ & $42 \pm 2$ & $54 \pm 1.5$ \\
$\mathrm{M}_{1}(\mathrm{M} \odot)$ & $7.70 \pm 0.66$ & $3.97 \pm 0.95$ & $7.24 \pm 0.70$ \\
Dist $(\mathrm{kpc})$ & $2.29 \pm 0.15$ & $2.49 \pm 0.30$ & $5.89 \pm 0.26$ \\
\hline Black Body $\dagger$ & $\mathrm{K}: 100 \%$ & $\mathrm{~K}: 71 \%$ & $\mathrm{~K}: 100 \%$ \\
& $3.5 \mu \mathrm{m}: 100 \%$ & $3.5 \mu \mathrm{m}: 38 \%$ & $3.5 \mu \mathrm{m}: 67 \%$ \\
Power Law* & $\mathrm{K}: 0 \%$ & $\mathrm{~K}: 29 \%$ & $\mathrm{~K}: 0 \%$ \\
& $3.5 \mu \mathrm{m}: 0 \%$ & $3.5 \mu \mathrm{m}: 62 \%$ & $3.5 \mu \mathrm{m}: 33 \%$ \\
Implication & No Disk or Jet & Synchrotron Jet & Weak Synchrotron Jet \\
\hline
\end{tabular}

* All LMXB parameters from Gelino, D. (2001) and all SED model results from this work.

$\dagger$ Contribution from the secondary star

** Contribution from a synchrotron jet
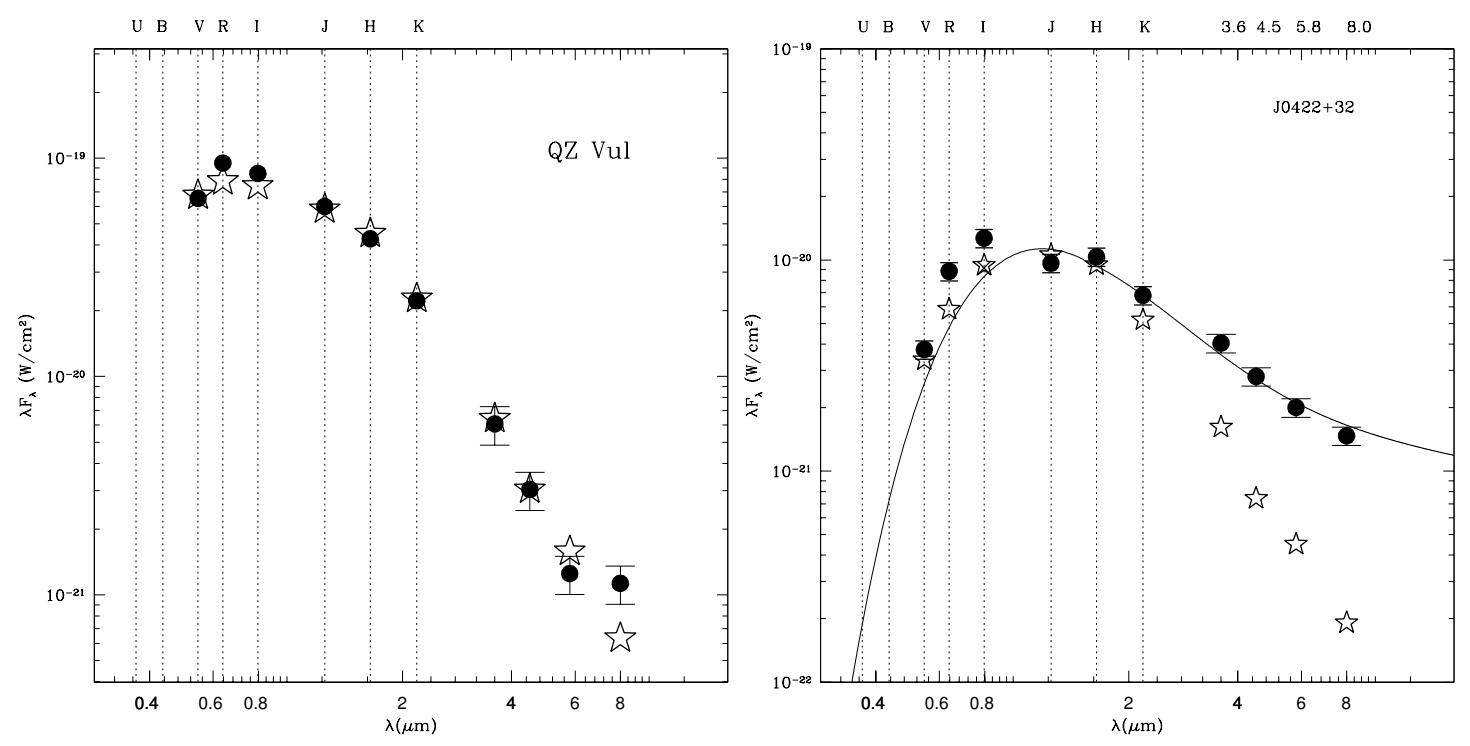

FIGURE 1. Spectral Energy Distributions (SEDs) for two LMXBs (filled circles). Error bars are $1 \sigma$ and are the size of the data points when not shown. Left: SED of QZ Vul dereddened by $A_{V}=3.78$ mag. Open stars represent the SED of a K2V. There does not appear to be any contaminating IR light from the system components. Right: SED of V518 Per (aka J0422+32) de-reddened by $A_{V}=0.25$ mag. Open stars represent the SED of a M1V. The solid line represents a cool blackbody model combined with a synchrotron power law that cuts off at $1.5 \mu \mathrm{m}$ simulating a jet in the system. The optical data were taken during an epoch of higher activity than the IR data and show the need for simultaneous observations. 


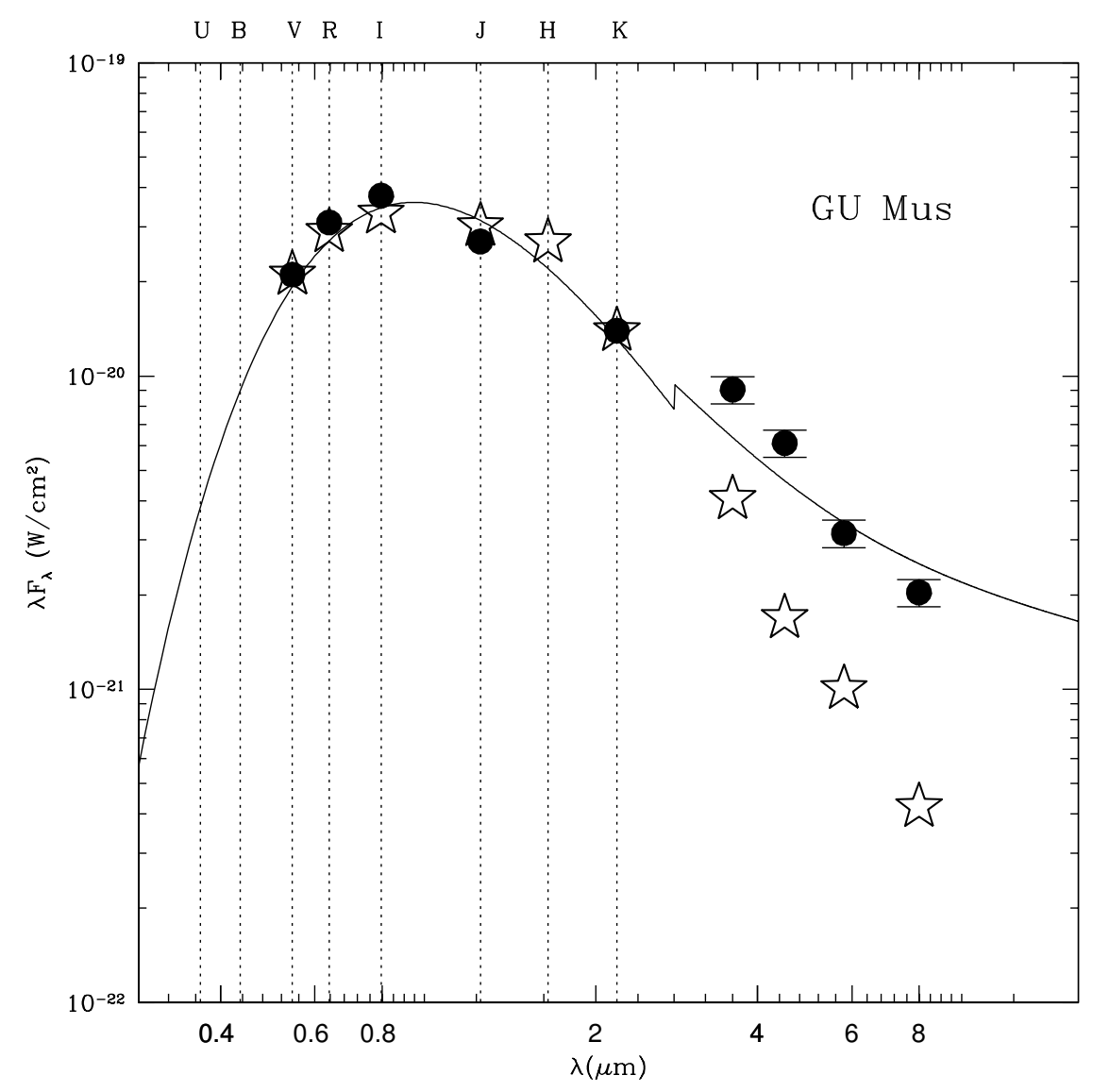

FIGURE 2. SED of GU Mus de-reddened by $A_{V}=0.9$ mag (filled circles). Error bars are as indicated in Figure 1. Open stars represent the SED of a K4IV (subgiant). The solid line represents a cool blackbody model combined with a synchrotron power law that cuts off at $2.8 \mu \mathrm{m}$ simulating a weak jet in the system. The optical and ground-based IR data were taken during an epoch of lower activity than the IR data (hence the break in the model) and show the need for simultaneous observations.

band light curves. Consequently, the K-band may not the best place to detect ellipsoidal variations from quiescent LMXBs. It is essential to combine ground-based and spacebased data, and thus Spitzer data is the key to determining the source of contaminating IR light in LMXBs. 